\title{
PROPAGATION OF EDGE WAVES IN A THINLY LAYERED LAMINATED MEDIUM WITH STRESS COUPLES UNDER INITIAL STRESSES
}

\author{
Lokenath Debnath \\ Department of Mathematics \\ University of Central Florida \\ Orlando, Florida 32816, U.S.A. \\ and \\ Pijush Pal Roy \\ Blasting Department \\ Central Mining Research Station \\ Barwa Road, Dhanbad - 826001, India
}

\begin{abstract}
The propagation of edge waves in a thinly layered laminated medium with stress couples under initial stresses is examined. Based upon an approximate representation of a laminated medium by an cquivalent anisotropic continuum with average initial and couple stresses, an explicit form of frequency equation is obtained to derive the phase velocity of edge waves. Edge waves exist under certain conditions. The inclusion of couple stresses increases the velocity of wave propagation. For a specific compression, the presence of couple stresses increases the velocity of wave propagation with the increase of wave number, whereas the reverse is the case when there is no couple stress. Numerical computation is performed with graphical representations. Several special cases are also examined.
\end{abstract}

Key words: Edge waves, composite elastic medium, frequency equation and instability.

1980 AMS Subject Classification Codes: 73D

\section{INTRODUCTION}

Considerable attention has been given to the specific features and propagation characteristic of edge waves in an elastic medium. These studies were recorded in several monographs including Love [1] and Ewing et al. [2] Subsequently, Kumar [3] made an elaborate study of the propagation of edge waves in homogeneous isotropic plates. Das and Dey [4] have extended Kumar's work by incorporating the effect of initial stresses in the governing equations. In spite of these studies, the propagation of edge waves in composite structures of thinly laminated materials has received little attention. However, 
such structures are found to have many geophysical applications. They are also used extensively in the study of buckling and vibrations.

Biot [5] provided an approximate representation of a laminated medium by a continuous structure with anisotropic properties. The assumptions required for the validity of such an approximate representation imply that the rigidity contrast of the layers is not too large, and that layer thicknesses must remain sufficiently small with respect to the wavelength of the deformation field. Based upon the averaging process, Biot [6] studied the folding problems of the first and the second kind, and showed that the same thing can be applied to the problem of the folding of a single anisotropic layer. Biot $[7,8]$ also offered the static and dynamic analyses of multilayered orthotropic elastic and viscoelastic plates which include the case in which anisotropy of the individual layer is due to a thinly laminated structure.

In a series of papers, Pal Roy [9], Pal Roy and Sinha [10], and Pal Roy and Debnath [11] have investigated the propagation characteristics of elastic waves in a layered laminated medium under initial and couple stresses. These studies also consider the dynamics of a laminated medium of Maxwell type solids and surface instability of a laminated material.

This paper is concerned with the propagation of edge waves in a thinly layered laminated medium with stress couples under initial stresses. This work is based on an approximate representation of a laminated medium by an equivalent anisotropic continuum with average initial and couple stresses. The explicit form of the frequency equation is determined so that the phase velocity of the edge waves can be calculated. Certain conditions among the couple stress coefficient, initial stresses, and the wave numbers ensure the existence of edge waves in the layered medium. The inclusion of couple stresses increases the velocity of wave propagation. For a specific compression, the presence of couple stresses increases the velocity of wave propagation with the increase of wave numbers, whereas the reverse is the case when there is no couple stress. Several special cases are also considered. Numerical computation is performed for a specific laminated structure of ten thin adhering layers with different elastic moduli. The graphical representation is given to show the importance and effects of couple stresses, initial stresses, and wave numbers. 


\section{THINLY LAYERED LAMINATED MEDIUM}

We consider a laminated medium that is made up of $n$ thin, alternating hard and soft layers. The $i$ th layer occupies a fraction $\alpha_{i}$ of the total thickness $H$. If the layers are sufficiently thin and the rigidity contrast of the layers is not too large, such a medium behaves like an elastic continuum with anisotropic properties, although the individual layers may be isotropic ( see Biot [5]). The $i$ th layer is specified by the elastic coefficients $N_{i}$ and $Q_{i}(i=1,2,3, \ldots n)$. We suppose that the $y$-axis is normal to the plane of the laminations. The stress-strain relations of the composite medium are given by (Biot [5], p. 186):

$$
s_{11}-s=2 N e_{x x}
$$

$$
\begin{aligned}
& s_{22}-s=2 N e_{y y} \\
& s_{12}=2 Q e_{x y}
\end{aligned}
$$

with the composite elastic coefficients $N$ and $Q$ where

$$
N=\sum_{i=1}^{n} \alpha_{i} \cdot N_{i} \text { and } \frac{1}{Q}=\sum_{i=1}^{n} \frac{\alpha_{i}}{Q_{i}} \text {. }
$$

The coefficients in (2.4ab) constitute a first approximation, whereas the influence of couple-stress provides a second approximation.

On the other hand, if the laminations are composed of isotropic materials, then we have

$$
N_{i}=Q_{i}=\lambda_{i}, \quad i=1,2, \ldots n
$$

The composite moduli are then 


$$
N=\sum_{i=1}^{n} \lambda_{i} \alpha_{i} \text { and } \frac{1}{Q}=\sum_{i=1}^{n} \frac{\alpha_{i}}{\lambda_{i}} .
$$

We now consider the influence of initial stresses in the composite structure. The principal directions are oriented along the $x$-axis and the $y$-axis. If $S_{11}^{(i)}$ and $S_{11}^{(i+1)}$ are the principal initial stresses along the laminations in the $i$ th and $(i+1)$ st layers respectively, then the average initial stress in the $x$-direction is given by

$$
S_{11}=\sum_{i=1}^{N} \alpha_{i} S_{11}^{(i)}
$$

The $y$-component of the initial stresses $S_{22}$ is constant throughout. In particular, when $S_{22}=0$, the quantities $p^{(i)}=-S_{11}^{(i)} \quad(i=1,2, \ldots n)$ represent compressive stresses in a direction parallel to the layers. The average compression is then

$$
P=\sum_{i=1}^{n} \alpha_{i} p^{(i)}
$$

\section{COUPLE STRESS ANALOGY}

It follows from the theory of Biot [7] that the use of an equivalent anisotropic continuum with equivalent elastic coefficients $N$ and $Q$ is not sufficient for good predictions. A better representation can be made by considering the bending rigidity of the laminations. According to Biot's analysis [7], the bending moment $C_{i}$ of the $i$ th layer of thickness $h_{i}$ is given by

$$
C_{i}=\frac{1}{3}\left(Q_{i}-Q\right) \frac{N_{i}}{Q_{i}} h_{i}^{3} \frac{\partial^{2} v}{\partial x^{2}}, \quad(i=1,2, \ldots n)
$$


The average bending moment $C$ of the equivalent anisotropic medium is obtained by extending the result (3.1) in each layer so that

$$
C=\frac{1}{H} \sum_{i=1}^{n} C_{i}=b \frac{\partial^{2} v}{\partial x^{2}}
$$

where

$$
b=\frac{1}{3} H^{2} \sum_{i}\left(1-\frac{Q}{Q_{i}}\right) N_{i} \cdot \alpha_{i}^{3}=\sum_{i} b_{i}
$$

and

$$
h_{i}=\alpha_{i} \cdot H
$$

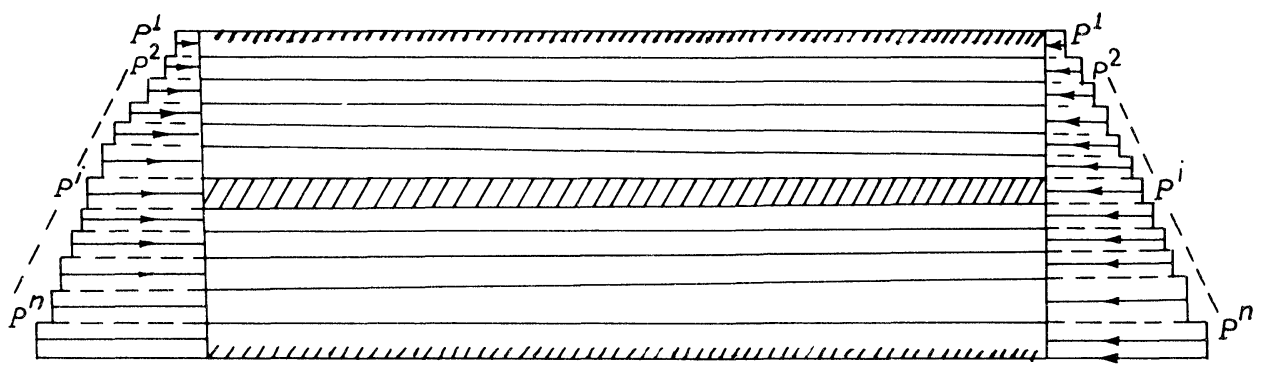

Fig. L: Thinly layered laminated plate of thickness $H$. 


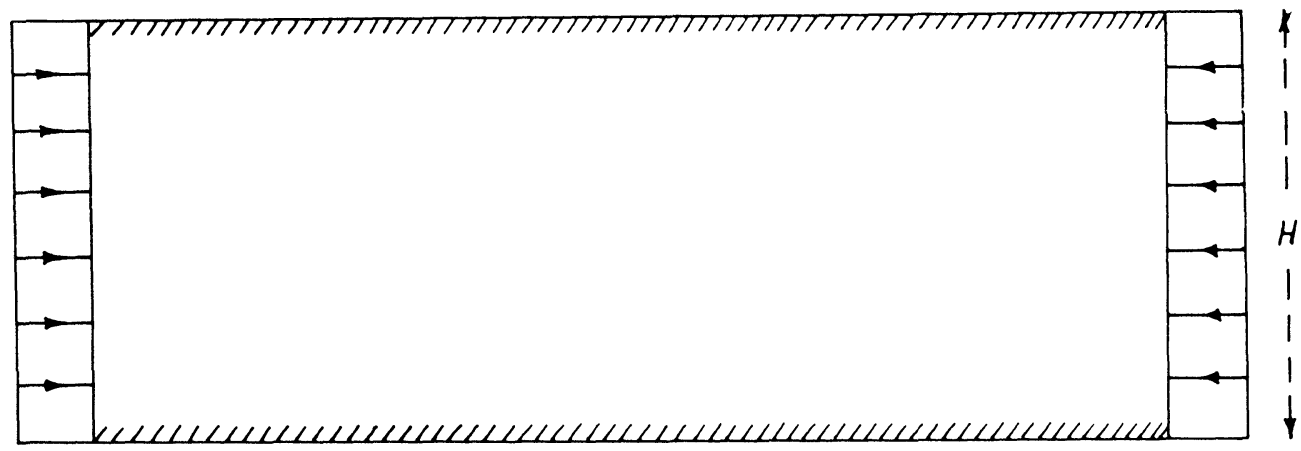

Fig. 2: Equivalent anisotropic plate under overage initial compression.

\section{FORMULATION OF THE PROBLEM}

We consider a thinly layered laminated plate of thickness $H$ and of infinite length under average initial compressive stress $P$ along the $x$-axis as shown in Figure 3.

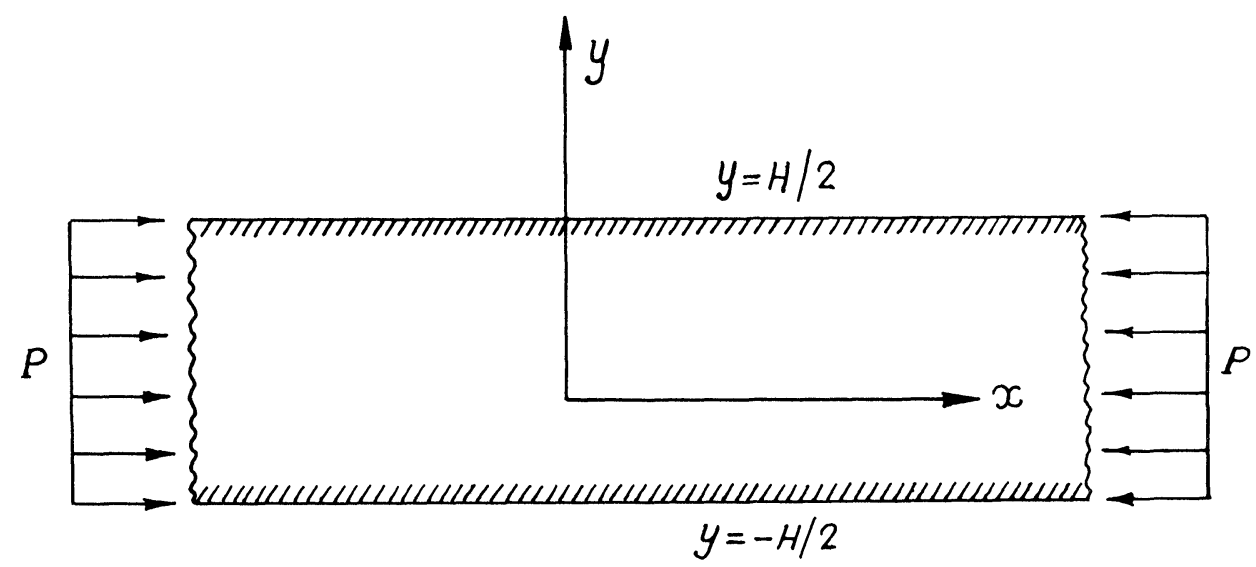

Fig. 3: An equivalent anisotropic plate of infinite length and thickness $H$, under initial compressive stress $P$. 
We shall restrict our discussion to the plane strain analysis only. The modified dynamical equations of equilibrium of the composite anisotropic medium are given by

$$
\begin{gathered}
\frac{\partial s_{11}}{\partial x}+\frac{\partial s_{12}}{\partial y}-P \frac{\partial \omega}{\partial y}=\rho \frac{\partial^{2} u}{\partial t^{2}} \\
\frac{\partial s_{12}}{\partial x}+\frac{\partial s_{22}}{\partial y}-P \frac{\partial \omega}{\partial x}=\rho \frac{\partial^{2} v}{\partial t^{2}}+b \frac{\partial^{4} v}{\partial x^{4}} .
\end{gathered}
$$

The condition of incompressibility is

$$
e_{x x}+e_{y y}=0
$$

The incremental stress-strain relations are

$$
\begin{gathered}
s_{11}-s=2 N e_{x x} \\
s_{22}-s=2 N e_{y y} \\
s_{12}=2 Q e_{x y}
\end{gathered}
$$

where

$$
s=\frac{1}{2}\left(s_{11}+s_{22}\right) \text {. }
$$

The equivalent elastic coefficients $N$ and $Q$ are given in relations (2.6ab)

\section{SOLUTION OF THE PROBLEM}

The condition of incompressibility (4.3) is satisfied by

$$
u=-\frac{\partial \psi}{\partial y} \text { and } v=\frac{\partial \psi}{\partial x}
$$

where $\psi=\psi(x, y, t)$. 
If we now use the relations (5.1ab) and (4.4abc), then equations (4.1) and (4.2) reduce to

(5.2a) $\frac{\partial s}{\partial x}-\left(Q+\frac{P}{2}\right) \frac{\partial}{\partial y}\left(\frac{\partial^{2} \psi}{\partial x^{2}}+\frac{\partial^{2} \psi}{\partial y^{2}}\right)+2(Q-N) \frac{\partial}{\partial y}\left(\frac{\partial^{2} \psi}{\partial x^{2}}\right)=-\rho \frac{\partial^{2}}{\partial t^{2}}\left(\frac{\partial \psi}{\partial y}\right)$

$$
\begin{aligned}
& \frac{\partial s}{\partial y}+\left(Q-\frac{P}{2}\right) \frac{\partial}{\partial x}\left(\frac{\partial^{2} \psi}{\partial x^{2}}+\frac{\partial^{2} \psi}{\partial y^{2}}\right)-2(Q-N) \frac{\partial}{\partial y}\left(\frac{\partial^{2} \psi}{\partial y^{2}}\right) \\
& =\rho \frac{\partial^{2}}{\partial t^{2}}\left(\frac{\partial \psi}{\partial x}\right)+b \frac{\partial^{5} \psi}{\partial x^{5}}
\end{aligned}
$$

Eliminating $s$ from the above two equations, we obtain

$$
\begin{aligned}
& (1-\delta) \frac{\partial^{4} \psi}{\partial x^{4}}+(1+\delta) \frac{\partial^{4} \psi}{\partial y^{4}}+2 L \frac{\partial^{4} \psi}{\partial x^{2} \partial y^{2}} \\
& =\frac{\rho}{Q} \frac{\partial^{2}}{\partial t^{2}}\left(\frac{\partial^{2} \psi}{\partial x^{2}}+\frac{\partial^{2} \psi}{\partial y^{2}}\right)+\frac{b}{Q} \frac{\partial^{6} \psi}{\partial x^{6}}
\end{aligned}
$$

where $\delta=P /(2 Q)$ and $L=(2 N / Q)-1$.

For harmonic waves along the $x$-direction, the solution of equation (5.3) is

$$
\psi=\frac{1}{m^{2}} g(m y) \exp [ \pm i(m x-\alpha t)]
$$

where $m=2 \pi / \lambda$ is the wave number and the function $g(m y)$ satisfies the equation

$$
g^{(i v)}(m y)-2 R g^{\prime \prime}(m y)+S^{2} g(m y)=0
$$

where apostrophes represent differentiation with respect to $m y$ and 


$$
\begin{aligned}
& W=\frac{\rho v^{2}}{Q(1+\delta)}, \frac{1-\delta}{1+\delta}=K^{2} \\
& 2 R=\frac{2 L}{(1+\delta)}-\frac{\rho v^{2}}{Q(1+\delta)}
\end{aligned}
$$

$$
\begin{aligned}
& T=\frac{b m^{2}}{Q(1+\delta)}, \quad V=\frac{\alpha}{m} \\
& S^{2}=K^{2}-W+T .
\end{aligned}
$$

As the propagation of pulse resembles the antisymmetric type of deflection, the solution of equation (5.5) is

$$
\psi=\frac{1}{m^{2}}\left(A_{1} \cosh \beta_{1} m y+A_{2} \cosh \beta_{2} m y\right) \sin (m x-\alpha t)
$$

where $\pm \beta_{1}$ and $\pm \beta_{2}$ are real or complex conjugate roots of the equation:

$$
\beta^{4}-2 R \beta^{2}+S^{2}=0 \text {. }
$$

Or equivalently

$$
\beta_{1}^{2}=R+\left(R^{2}-S^{2}\right)^{1 / 2}
$$

$$
\beta_{2}^{2}=R-\left(R^{2}-S^{2}\right)^{1 / 2}
$$

with conditions

(i) $R>0$, and $S^{2}>0$

or

(ii) $R<0$, and $\left(R^{2}-S^{2}\right)<0$. 
Now using the first of equations (5.2) and solution (5.10), we obtain

$$
\begin{aligned}
s= & \left(Q+\frac{P}{2}\right)\left[g^{\prime}(m y)-g^{\prime \prime}(m y)\right] \cos (m x-\alpha t) \\
& -\left[\rho \frac{\alpha^{2}}{m^{2}}+2(Q-N)\right] g^{\prime}(m y) \cos (m x-\alpha t) .
\end{aligned}
$$

\section{BOUNDARY CONDITIONS}

The boundary conditions of the problem are concerned with the bounding planes of the composite anisotropic medium and are supposed to be free from tractions. The boundary conditions as suggested by Biot [5] are

$$
\Delta f_{x}=s_{12}+P e_{x y}=0
$$

$$
\text { at } y= \pm H
$$

$$
\Delta f_{y}=s_{22}=0
$$

where $\Delta f_{x}$ and $\Delta f_{y}$ are incremental forces per unit of initial area at the boundary. Using the expression for $\psi$ and the stress-strain relations $(2.1)-(2.3)$, we allow the conditions (6.1 ab) to reduce to

$$
A_{1}\left(1+\beta_{1}^{2}\right) \cosh \beta_{1}\left(\frac{m H}{2}\right)+A_{2}\left(1+\beta_{2}^{2}\right) \cosh \beta_{2}\left(\frac{m H}{2}\right)=0
$$

$$
A_{1} \beta_{1}\left(2 R+1-\beta_{1}^{2}\right) \sinh \beta_{1}\left(\frac{m H}{2}\right)+\beta_{2} A_{2}\left(2 R+1-\beta_{2}^{2}\right) \sinh \beta_{2}\left(\frac{m H}{2}\right)=0 \text {. }
$$

Eliminating the constants $A_{1}$ and $A_{2}$ from equations (6.2), we obtain

$$
\beta_{2}\left(1+\beta_{1}^{2}\right)^{2} \tanh \left(\beta_{2} \lambda\right)-\beta_{1}\left(1+\beta_{2}^{2}\right)^{2} \tanh \left(\beta_{1} \lambda\right)=0
$$

where $\lambda=m H / 2$.

This equation determines the phase velocity $V=\alpha / m$ of the edge waves in the composite structure when $\rho, N, Q$ and $\lambda$ are specified. It is clear that the waves are 
dispersive in nature. The phase velocity depends on $\delta$ and hence on the average initial compression $P$. The relation between $V$ and $\delta$ can be established for particular values of the parameters. The velocity depends on $N$ and $Q$, which in turn depend on the initial stress. The velocity also depends on $b$, the average couple-stress coefficient of the equivalent continuum.

\section{SOME PARTICULAR CASES}

Let us consider as a first case the situation wherein the total thickness $H$ of the composite structure is itself small. In this situation

$$
\tanh \left(\beta_{1} \lambda\right) \rightarrow \beta_{1} \lambda \text { and } \tanh \left(\beta_{2} \lambda\right) \rightarrow \beta_{2} \lambda
$$

The frequency equation (6.3) reduces to

$$
\beta_{1}^{2} \beta_{2}^{2}=1
$$

Because of relations (5.6) - (5.9) and (5.12ab), equation (7.2) ultimately reduces to the form

$$
V^{2}=\frac{b m^{2}-P}{\rho}
$$

Three conditions may now arise.
(i) $b m^{2}>P, b \neq 0$
(ii) $b m^{2}<P, b \neq 0, P \neq 0$
(iii) $b=0, P \neq 0$

Condition (i) ensures the existence of edge waves under the initial compression in a layered laminated structure. However, conditions (ii) and (iii) do not allow propagation of edge waves under initial stresses. This conclusion is in agreement with the result of Biot [5] for 
the case of a homogeneous isotropic elastic medium. When condition (ii) or (iii) is satisfied, surface instability ensues in the medium as soon as small compression is applied so that waves do not propagate under either of the two conditions. Thus the existence of edge waves is solely due to couple stresses involved in (i).

For a second case, let us assume

$$
\begin{aligned}
& \tanh \beta_{1} \lambda \sim \beta_{1} \lambda-\frac{1}{3} \beta_{1}^{3} \lambda^{3} \\
& \tanh \beta_{2} \lambda \sim \beta_{2} \lambda-\frac{1}{3} \beta_{2}^{3} \lambda^{3}
\end{aligned}
$$

The frequency equation (6.3) reduces to the simple form

$$
\frac{3}{2 \lambda^{2}}=\frac{R+S^{2}}{1-S^{2}}
$$

Using the expressions for $R$ and $S$ as given in relations (5.6) - (5.9), we get the expression for $V$ as

$$
V^{2}=\frac{2 Q(L+1-\delta) \lambda^{2}+2 b m^{2} \lambda^{2}+3 b m^{2}-6 Q \delta}{3 \rho\left(1+\lambda^{2}\right)}
$$

This expression determines the velocity of edge waves in the equivalent anisotropic medium. Clearly, it depends on the average initial compression $P$ and the average couplestress coefficient $b$. The effect of $b$ on $V$ can be determined for specific values of other parameters.

\section{NUMERICAL CALCULATIONS AND DISCUSSIONS}

We now consider a laminated plate constituted by an alteration of ten thin adhering layers with different elastic moduli, in which the first and sixth layers are isotropic in nature. The following dimensions are taken into account: 


$$
\begin{aligned}
& H=10 \mathrm{~cm}, \\
& \delta=P / 2 Q=0,0.02,0.10,0.30,0.48,0.64,0.75, \\
& \lambda=0.1,0.2,0.3,0.4,0.5 \mathrm{~cm}, \\
& \rho=\sum_{i=1}^{10} \alpha_{i} \rho_{i}=2.3 \mathrm{gm} / \mathrm{cm}^{3}
\end{aligned}
$$

\begin{tabular}{|c|c|c|c|c|}
\hline laycr \# & $Q_{i}\left(10^{11}\right.$ dyncs $\left./ \mathrm{cm}^{2}\right)$ & $N_{i}\left(10^{11}\right.$ dynes $\left./ \mathrm{cm}^{2}\right)$ & $\alpha_{i}, \Sigma \alpha_{i}=1$ & $h_{i}, \Sigma h_{i}=H=10 \mathrm{~cm}$ \\
\hline 1 & 1.6 & 1.6 & 0.1111111 & 1.111111 \\
\hline 2 & 1.7 & 2.7 & 0.0666666 & 0.666666 \\
\hline 3 & 1.8 & 5.7 & 0.05 & 0.5 \\
\hline 4 & 2.01 & 2.04 & 0.1666666 & 1.666666 \\
\hline 5 & 1.4 & 2.8 & 0.076923 & 0.76923 \\
\hline 6 & 1.2 & 1.2 & 1.125 & 1.25 \\
\hline 7 & 2.15 & 3.10 & 0.1 & 1 \\
\hline 8 & 1.9 & 4.2 & 0.090909 & 0.90909 \\
\hline 9 & 1.15 & 2.10 & 0.0833333 & 0.833333 \\
\hline 10 & 3.10 & 4.11 & 0.1293904 & 1.293904 \\
\hline
\end{tabular}

$$
\begin{gathered}
Q=1.7034936 \times 10^{11} \text { dynes } / \mathrm{cm}^{2}, N=2.7467741 \times 10^{11} \mathrm{dyncs} / \mathrm{cm}^{2} \\
b / Q=0.0876765[\text { Eqn. (3.3) ] } \quad[\text { Eqns. }(2.4 \mathrm{ab})] .
\end{gathered}
$$

In the first case of Section 7, it is found when $b \neq 0$ that edge waves exist for $P=($; and instability will occur at once if a certain amount of compression begins. When $b=()$, there is no wave propagation in the compression-free case; and if a certain amount of compression is applied, instability will occur at once.

In the second case of Section 7 , the values of $V^{2} / Q$ are calculated from equation (7.6) against the instability factor $\delta=P / 2 Q$ and plotted to exhibit the following graph: 


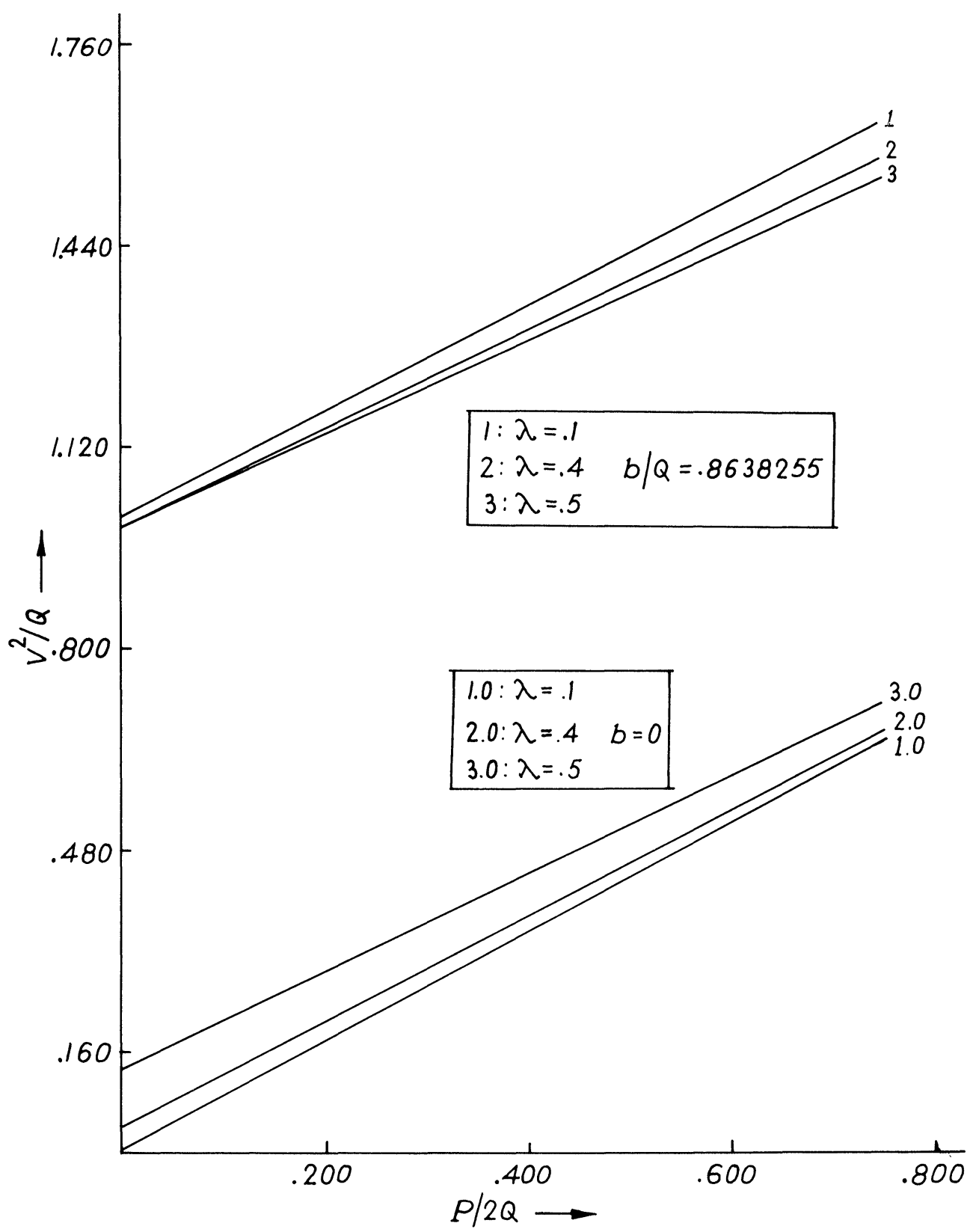

Fig 4: $V^{2} / Q$ as a function of the stability parameter $\delta(=P / 2 Q)$ ploted for three values of $\lambda$ with and without taking couple stress effect. 
The following conclusions can be drawn from Fig. 4.

(i) The increase in $\lambda$ (hence the wave number) brings about a decrease in $V$ when $b \neq 0$.

(ii) While $\lambda$ increases, the velocity $(v)$ increases when $b=0$.

(iii) The inclusion of couple stress $(b)$ increases the velocity of wave propagation.

(iv) When there is a couple stress, then for a specific compression, the velocity increases as $\lambda$ increases; but the converse is the case when there is no couple stress.

Acknowledgement: The authors express their grateful thanks to Dr. S. Dey of the Indian School of Mines at Dhanbad for his help and interest in this work. The second author is thankful to Jadavpur University for providing facilities under the UGC-DSA Program in 1985. This research was partially supported by the University of Central Florida.

\section{REFERENCES}

[1] Love, A. E. H., A Treatise on the Mathematical Theory of Elasticity, Dover, New York, 1944.

[2] Ewing, M.A., Jardetsky, W.S. and Press, F., Elastic Waves in Layered Media, McGraw-Hill, New York, 1957.

[3] Kumar, S., Edge Waves in Plates, Int. Symp. on Stress Wave Propagating in Materials, Pennsylvania State University, U.S.A., 1959.

[4] Das, S.C. and Dey, S., Edge Waves Under Initial Stress, Appl. Sci. Res. 22 (1970), pp. 382-389. 
[5] Biot, M.A., Mechanics of Incremental Deformations, John Wiley and Sons, New York, 1965.

[6] Theory of Similar Folding of the First and Second Kind, Geol. Soc. Am. Bull., 76 (1965), pp. 251-258.

[7] A New Approach to the Mechanics of Orthotropic Multilayered Plates, Int. J. Solids Struct., 8 (1972), pp. 475-490.

[8] Simplified Dynamics of Multilayered Orthotropic Viscoelastic Plates, Int. J. Solids Struct., 8 (1972), pp. 491-509.

[9] Pal Roy, P., Wave Propagation in a Thinly Two-layered Laminated Medium with Stress Couples Under Initial Stresses, Acta Mech., 54 (1984), pp. 1-21.

[10] Pal Roy, P. and Sinha, D.K., Surface Instability of a Thinly Layered Laminated Medium with Stress Couples Under Initial Stress, Proc. INSA, Part A, Physical Sciences. 53(A), No. 3 (1987), 399-408.

[11] Pal Roy, P. and Debnath, L., Dynamics of Multilayered Orthotropic Viscoelastic Plates Made up of Maxwell Solids, Internat. J. and Math. Sciences, 11 (1988), 177-186. 


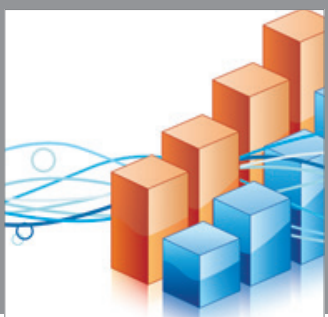

Advances in

Operations Research

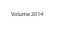

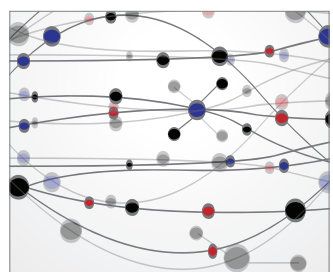

\section{The Scientific} World Journal
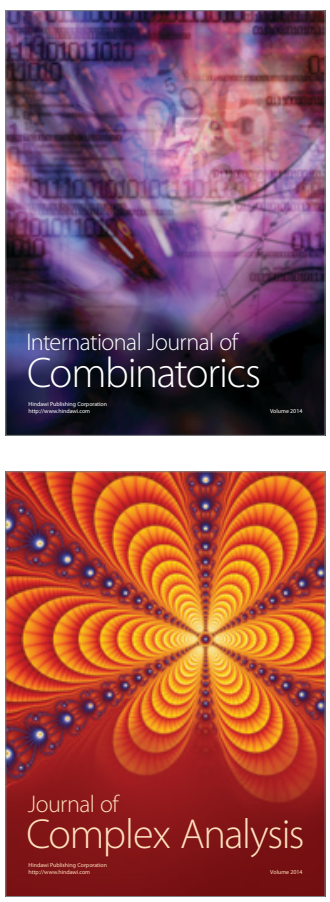

International Journal of

Mathematics and

Mathematical

Sciences
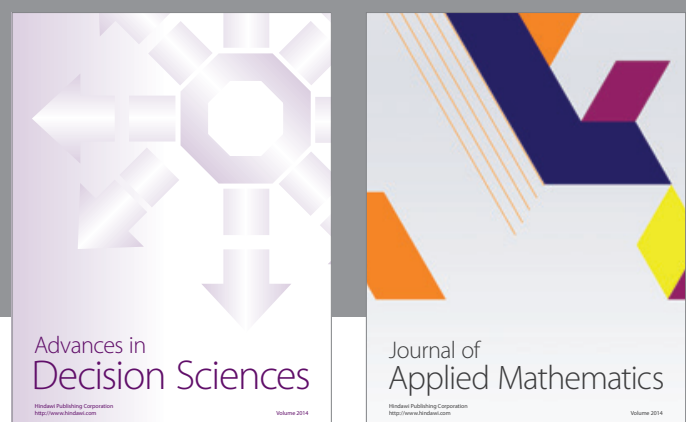

Journal of

Applied Mathematics
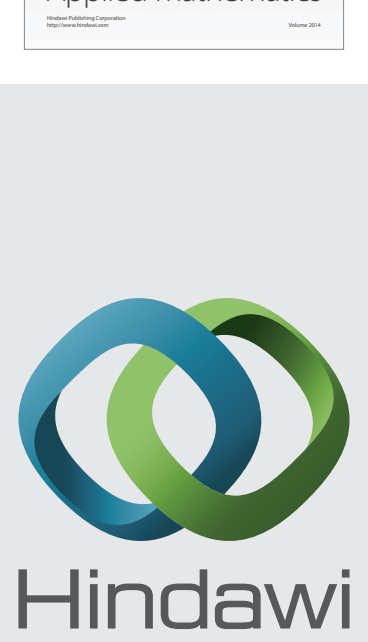

Submit your manuscripts at http://www.hindawi.com
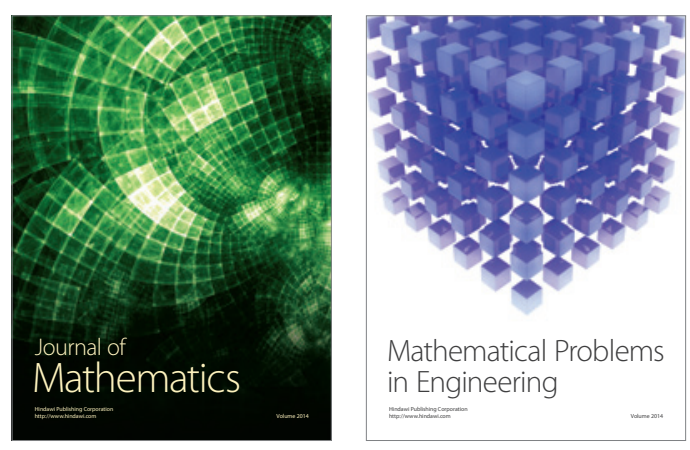

Mathematical Problems in Engineering
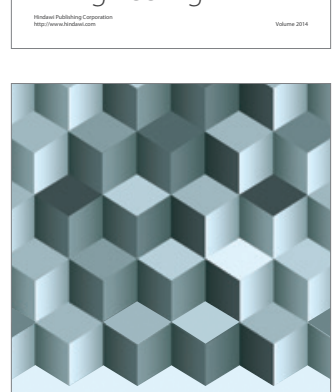

Journal of

Function Spaces
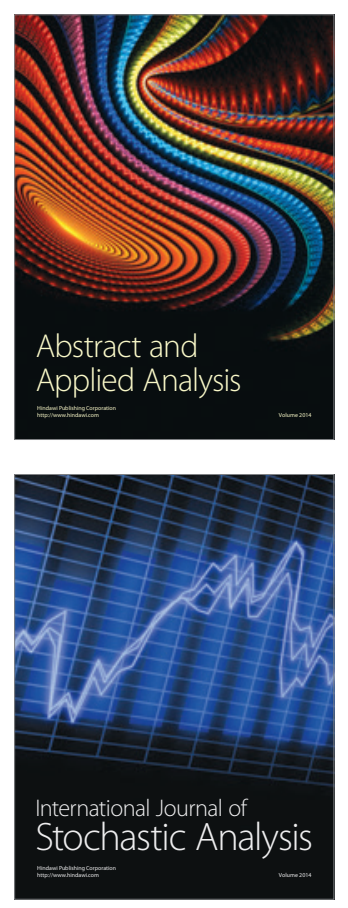

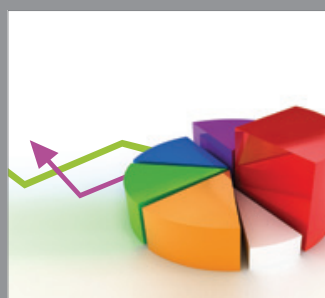

ournal of

Probability and Statistics

Promensencen
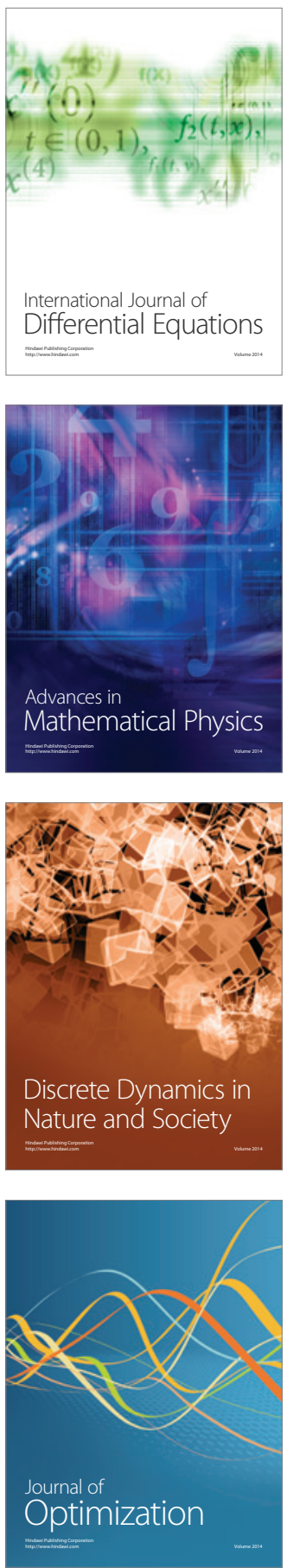